\title{
Rosuvastatin intensifies the beneficial effects of rho-kinase inhibitor in reversal of monocrotaline- induced pulmonary hypertension
}

\author{
Magdalena Jasińska-Stroschein, Jacek Owczarek, Urszula Sołtysiak, Daria Orszulak-Michalak
}

Department of Biopharmacy, Medical University of Lodz, Lodz, Poland

Submitted: 22 September 2014

Accepted: 24 November 2014

Arch Med Sci 2016; 12, 4: 898-905

DOI: $10.5114 /$ aoms.2015.49740

Copyright @ 2016 Termedia \& Banach

\section{Abstract}

Introduction: It remains controversial whether statins have a beneficial effect on pulmonary arterial hypertension (PAH). This study is intended to evaluate whether statin, co-administered with Rho-kinase inhibitor, could enhance its efficacy. Although Rho-kinase inhibitors, including fasudil, have been reported to improve pulmonary hypertension in experimental and clinical studies, the combination of these agents has not been tested in the treatment of pulmonary hypertension (PH).

Material and methods: The effects of such a regimen on hemodynamics, right ventricle hypertrophy, and Rho-associated protein kinase (ROCK) activity in experimental monocrotaline (MCT)-induced pulmonary hypertension were examined. Fourteen days after monocrotaline injection $(60 \mathrm{mg} / \mathrm{kg})$, male rats were treated orally for another 14 days with fasudil $(15 \mathrm{mg} / \mathrm{kg}$ per day), or with a combination of fasudil + rosuvastatin ( $10 \mathrm{mg} / \mathrm{kg}$ per day).

Results: The drug combination reversed the MCT-induced increase in right ventricle pressure (RVP) and reduced right ventricular hypertrophy (RV/LV + $\mathrm{S}$ ratio) more than Rho kinase inhibitor alone. The simultaneous administration of fasudil and rosuvastatin caused a further decrease of RhoA kinase activity in isolated lung tissues as compared to fasudil alone.

Conclusions: The results indicate that rosuvastatin intensifies the beneficial effects of Rho-kinase inhibitor on the Rho/Rho-kinase pathway and such a combination may represent an option for the treatment of pulmonary arterial hypertension.

Key words: pulmonary hypertension, fasudil, rosuvastatin, combination therapy, Rho/Rho kinase pathway.

\section{Introduction}

Pulmonary arterial hypertension (PAH) is a multifactorial disease initially manifesting as an abnormal elevation in pulmonary arterial pressure (PAP) and pulmonary vascular resistance (PVR), followed by endothelial cell dysfunction, vascular proliferation and pulmonary arteriolar remodeling [1, 2].

Recent evidence has shown that Rho-kinases (ROCKs) have a preponderant role in the physiopathology of pulmonary hypertension ( $\mathrm{PH}$ ) due to their potent effects on pulmonary arterial smooth muscle cell (SMC) contraction and proliferation [3, 4]. The role of the RhoA/Rho-kinase signaling pathway in the pathogenesis of $\mathrm{PH}$ has been confirmed in diverse animal models, including monocrotaline (MCT)-induced $\mathrm{PH}$ [5].

\author{
Corresponding author: \\ Magdalena Jasińska- \\ Stroschein MD \\ Department of Biopharmacy \\ Medical University of Lodz \\ 1 Muszyńskiego St \\ 90-151 Lodz, Poland \\ Phone: +48 426779121 \\ E-mail: magdalena.jasinska@ \\ umed.lodz.pl
}


The monocrotaline pyrrole is activated in the liver and its metabolites cause direct endothelial damage that leads to the development and progression of severe/lethal pulmonary hypertension. Injection of MCT induces proliferative vasculitis, vascular remodeling, myocarditis and right ventricle hypertrophy [6]. The progression of $\mathrm{PH}$ is associated with pathological changes in pulmonary arteries; myocardial hypertrophy is a compensatory mechanism whereby cardiac tissue adapts to increased workload after monocrotaline treatment [7]. One should consider, however, that such a model might not fully represent pulmonary hypertension in humans seen in daily clinical practice and being the consequence of various pathologies.

The beneficial effects of ROCK inhibitors have been demonstrated in several experimental studies [8] as well as some short- and mid-term clinical trials. They included patients with pulmonary hypertension of different origin: idiopathic, $\mathrm{PAH}$ associated with connective tissue disease or congenital heart disease [9-11].

Long-term inhibition of Rho-kinase with fasudil (HA-1077) ameliorated rat monocrotaline (MCT)-induced $\mathrm{PH}$ as well as mice hypoxia-induced $\mathrm{PH}[12,13]$. It has also been demonstrated to have favorable short-term effects in patients with $\mathrm{PH}$. Also, the results of a very recent double-blind, placebo-controlled clinical trial indicate the benefits of mid-term, oral treatment with this drug. Such treatment showed additional improvement of $\mathrm{PAH}$ including pulmonary hemodynamics and cardiac index [14]. Fasudil is the first approved ROCK for clinical use in the treatment of ischemia-induced brain damage [15].

HMG-CoA reductase inhibitors (statins) are well known for their pleiotropic effects, which go beyond serum cholesterol lowering activity, such as cardiovascular effects. These include improving or restoring endothelial function, as well as inhibiting the proliferation and migration of vascular inflammation and oxidative stress [16, 17]. Several studies have aimed to assess their role in pulmonary arterial hypertension treatment. However, in contrast to ROCK inhibitors, statins' advantages in disease improvement seem to remain controversial. In several experimental animal models, statins have been reported to exert additive effects when combined with conventional agents in $\mathrm{PH}[18,19]$. In previous clinical trials statin has revealed no evident (APATH study) or transient efficacy when added to conventional agents in $\mathrm{PH}$, i.e. sildenafil and/or endothelin-1 antagonist (SIPHT study), and to date, the advantageous role of statins has not been confirmed definitely $[20,21]$.

Our previous experimental results [22] show that rosuvastatin may exert only a moderate im- pact on hemodynamics or myocardial hypertrophy improvement in an MCT rat model. According to our knowledge, the effects of a combined statin and Rho kinase inhibitor regimen have not been assessed in $\mathrm{PH}$. A very recent study described the synergism of concurrent administration of another statin, pitavastatin, and fasudil in a rabbit model of subarachnoid hemorrhage [23]. Hence, considering previous promising reports on interaction between statin and Rho kinase inhibitor, the aim of the study was to evaluate whether statin added to fasudil therapy provides added effects in $\mathrm{PH}$. In order to assess such potential effects, an experimental study was performed using a monocrotaline (MCT)-induced $\mathrm{PH}$ model in rats.

\section{Material and methods}

\section{Animals}

The experiments were performed on 32 young, 8- to 10-week-old, outbred Wistar male rats, fed on Murigran with free access to water. The animals were housed in standard cages. The experimental procedures were carried out in accordance with the international guidelines for care and use of laboratory animals. All efforts were made to minimize animal suffering and reduce the number of animals used in the experiments. All the procedures in these experiments were approved by the Ethics Committee of the Medical University of Lodz, Poland (48/ŁB 565/2011; 17/ŁB 602/2012; 67/ŁB 636/2012).

\section{Drugs}

Fasudil (HA-1077, Dihydrochloride Salt, Fluorochem Ltd, UK), and rosuvastatin were administered by oral gavage, suspended in $1.0 \%$ methylcellulose $(M C)$.

\section{Monocrotaline treatment}

Monocrotaline, MCT (Fluorochem Ltd, UK) was dissolved in $1 \mathrm{~N} \mathrm{HCl}$, and the $\mathrm{pH}$ was adjusted to 7.4 with $1 \mathrm{~N} \mathrm{NaOH}$ [24]. Monocrotaline was administered as a single, subcutaneous (s.c.) injection $(60 \mathrm{mg} / \mathrm{kg})$ in a volume of $3 \mathrm{ml} / \mathrm{kg}$. Control, agematched rats received an equal volume of vehicle.

\section{Experimental design}

The animals were divided into four groups of rats, randomly allocated to every group: 1 ) healthy rats receiving $1.0 \%$ methylcellulose (MC), $1 \mathrm{ml} / \mathrm{kg}$ bw, i.g. daily for 14 days - Sham; 2) MCT-induced rats receiving $1.0 \% M C, 1 \mathrm{ml} / \mathrm{kg}$ bw, i.g. daily for 14 days after single, subcutaneous MCT injection - MCT + Vehicle; 3) MCT-induced rats receiving fasudil, $15 \mathrm{mg} / \mathrm{kg}$ bw, i.g. daily for 14 days; 4) MCT-induced rats receiving fasudil, $15 \mathrm{mg} / \mathrm{kg}$ 
bw + rosuvastatin, $10 \mathrm{mg} / \mathrm{kg}$ bw, i.g. daily for 14 days. Rats were weighed once a week. The first dose of drug was given 14 days after MCT injection. The whole protocol lasted 29 days.

\section{Hemodynamic studies}

The surgery was performed $24 \mathrm{~h}$ after the administration of the last drug dose. The rats were placed in the dorsal position on the operating table. The animals were anaesthetized with pentobarbital sodium (30 mg/kg bw) [7], administered intraperitoneally. Anesthesia was maintained by additional bolus doses of pentobarbital sodium (15 mg/kg bw) as needed. Lidocaine was used for local infiltration of the surgical sites. The registration of hemodynamic parameters including mean right ventricle pressure (RVP), systolic, mean and diastolic arterial blood pressure (SABP, MABP, $D A B P)$ and heart rate (HR) in rats was performed using a Hugo Sachs Electronik Haemodyn (Harvard Apparatus GmbH, March, Germany), as described previously [25].

\section{Measurement of organ weight}

At the end of the study, after animal euthanasia, the heart was excised. Next, the right ventricle, right atrium and left ventricle plus septum were separated and weighed. The ratio of the right ventricle weight to left ventricle plus septum weight $(\mathrm{RV} / \mathrm{LV}+\mathrm{S})$ was calculated as an index of right ventricular hypertrophy.

\section{ROCK activity assay}

In order to measure ROCK activity, a commercially available kit (STA-416, 96-well ROCK activity

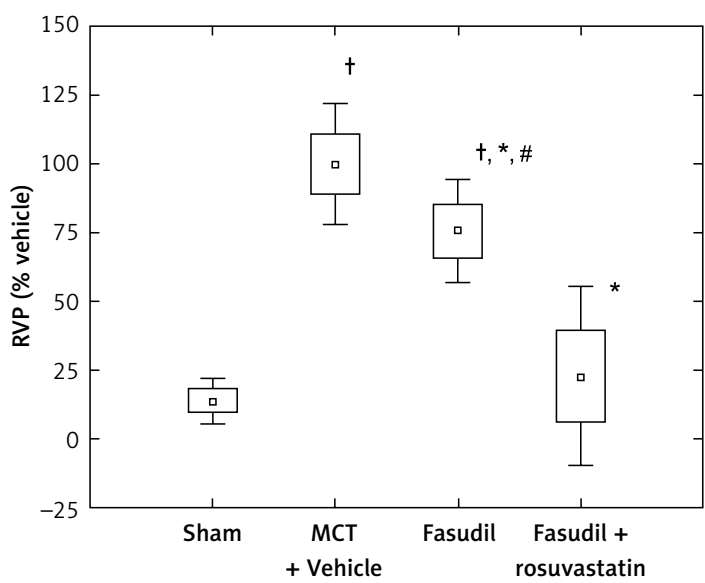

Figure 1. Right ventricle pressure (RVP) as percentage of MCT + Vehicle. Sham $(n=8) ;$ MCT + Vehicle $(n=9)$; fasudil $(n=6)$; fasudil + rosuvastatin $(n=8)$

The histograms indicate: mean $\pm S D(\square)$ and mean \pm $1.96{ }^{\star} S D(\perp) ;{ }^{*} p<0.0005$ vs. MCT + Vehicle; ${ }^{\dagger} p<0.0005$ vs. Sham; ${ }^{*} p<0.0005$ vs. combination group. assay kit, Cell Biolabs, San Diego, USA) was used according to the manufacturer's instructions. In brief, lung tissues were homogenized, and RIPA buffer (Sigma) was added. Subsequently, the samples were centrifuged at $12000 \mathrm{~g}$ for $10 \mathrm{~min}$ at $4^{\circ} \mathrm{C}$, and supernatants were collected and stored at $-80^{\circ} \mathrm{C}$ until further use. Each sample was assayed in duplicate. They were loaded in a microplate and the kinase reaction started by adding kinase reaction buffer. After 45 min incubation and washing, the wells were incubated with anti-phospho-MYPT1 (Thr696) antibody (1: 1000). After $1 \mathrm{~h}$, the wells were washed and HRP-conjugated secondary antibody was added for another hour. Afterwards, substrate solution was added and 20 min incubation followed. The enzyme reaction was stopped by adding Stop Solution. Active ROCK-Il served as a positive control. Absorbance was read using $450 \mathrm{~nm}$ (OD 450) as the primary wavelength.

\section{Endothelial nitric oxide synthase (eNOS) assay}

The examination of concentration of synthase for nitric oxide (eNOS) in lung tissues was performed using a commercially available kit (Life Science Inc., USA) according to the manufacturer's instructions. Lung tissue homogenates were prepared as described above.

\section{Statistical analysis}

The statistical analysis was carried out using Statistica version 10.0. The statistical evaluation was performed using the analysis of variance (ANOVA), and post-hoc comparisons were performed using the Student-Newman-Keuls test. Normal distribution of a parameter was checked by means of the Shapiro-Wilk test. The homogeneity of variance was tested by the Brown-Forsythe test. If the data were not normally distributed or the values of variance were different, ANOVA with the Kruskal-Wallis test were used. All parameters were considered statistically significantly different if $p<0.05$.

\section{Results}

\section{Animal survival}

By the end of the experiments, no rats had died in the control group or in the monocrotaline or other experimental groups.

\section{Hemodynamic studies}

Figure 1 shows the data for the right ventricle pressure (RVP), expressed as percentage of MCT + Vehicle rats. The mean RVP values in the MCT + Vehicle group were significantly increased com- 
Table I. Heart rate (HR), systolic; mean and diastolic blood pressure (SABP, MABP, DABP). Sham $(n=8) ;$ MCT + Vehicle $(n=9)$; fasudil $(n=6)$; fasudil + rosuvastatin $(n=8)$

\begin{tabular}{|lcccc|}
\hline Animals & HR $[\mathrm{bpm}]$ & SABP $[\mathrm{mm} \mathrm{Hg}]$ & MABP $[\mathrm{mm} \mathrm{Hg}]$ & DABP $[\mathrm{mm}$ Hg] \\
\hline Sham & $358.1 \pm 24.6$ & $99.2 \pm 3.2$ & $93.3 \pm 4.9$ & $87.8 \pm 6.0$ \\
\hline MCT + Vehicle & $402.6 \pm 27.8$ & $101.1 \pm 5.7$ & $95.9 \pm 5.7$ & $90.9 \pm 5.6$ \\
\hline Fasudil & $398.1 \pm 44.9$ & $95.0 \pm 8.3^{\#}$ & $93.0 \pm 8.1^{\#}$ & $89.3 \pm 8.1$ \\
\hline Fasudil + rosuvastatin & $374.0 \pm 85.4$ & $87.5 \pm 1.6^{*},+$ & $85.1 \pm 2.8^{*},+$ & $83.5 \pm 2.8^{*}$ \\
\hline
\end{tabular}

Data expressed as mean $\pm S D,{ }^{\dagger} p<0.05$ vs. Sham; ${ }^{*} p<0.05$ vs. MCT + Vehicle; ${ }^{*} p<0.05$ vs. combination group.

pared to Sham ( $p<0.0005)$. 14-day administration of fasudil, a Rho-kinase inhibitor, at a dose of $15 \mathrm{mg} / \mathrm{kg}$ bw, reduced RVP significantly compared to the MCT-treated animals ( $p<0.0005)$. Combined administration of fasudil and rosuvastatin caused a significant decrease in the RVP parameter compared to MCT $(p<0.0005)$ or fasudil alone $(p<0.0005)$ (Figure 1). Rosuvastatin given together with fasudil caused a significant decrease of arterial blood pressure compared to monocrotaline, fasudil alone or saline $(p<0.05)$. No significant differences regarding heart rates among examined groups of animals were observed (Table I).

\section{Cardiac hypertrophy}

Figure 2 shows the data for the ratio of the right ventricle weight to left ventricle plus septum weight $(\mathrm{RV} / \mathrm{LV}+\mathrm{S})$, expressed as percentage of $M C T+$ Vehicle. The RV/LV + S parameter was significantly higher in MCT-treated animals than in Sham $(p<0.0005)$. Fasudil administration caused a significant reduction of RV/LV $+\mathrm{S}$ as compared to MCT + Vehicle $(p<0.05)$. Combined administra-

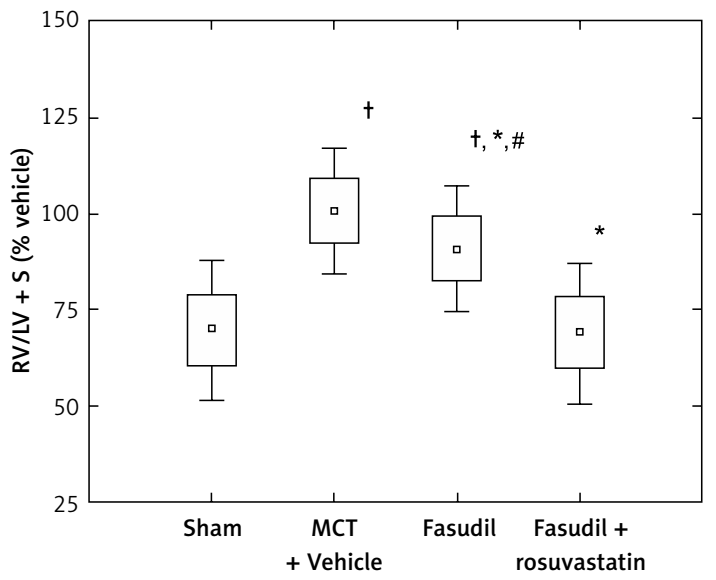

Figure 2. Right ventricle hypertrophy expressed as the ratio of the right ventricular weight to left ventricle plus septum weight $(\mathrm{RV} / \mathrm{LV}+\mathrm{S})$, as percentage of MCT + Vehicle. Sham $(n=8) ;$ MCT + Vehicle $(n=9)$; fasudil $(n=6)$; fasudil + rosuvastatin $(n=9)$

The histograms indicate: mean $\pm S D(\square)$ and mean \pm $1.96^{\star} S D(\perp) ;{ }^{*} p<0.05$ vs. MCT + Vehicle; ${ }^{\dagger} p<0.0005$ vs. Sham; $p<0.0005$ vs. combination group. tion of fasudil and rosuvastatin resulted in a significant decrease in $\mathrm{RV} / \mathrm{LV}+\mathrm{S}$ ratio compared to rats treated with MCT $(p<0.0005)$ or fasudil alone $(p<0.0005)$.

\section{ROCK activity evaluation}

Figure 3 shows the data for ROCK activity expressed as percentage of MCT + Vehicle. The ROCK activity was significantly higher in rat lung tissues with induced pulmonary arterial hypertension compared to the Sham $(p<0.0005)$. Fasudil administration tended to reduce ROCK activity compared to MCT $(p<0.0005)$. The simultaneous administration of fasudil and rosuvastatin caused a significant decrease of the parameter compared to MCT injection $(p<0.0005)$, fasudil alone $(p<0.005)$ and saline $(p<0.05)$.

\section{eNOS concentration evaluation}

Figure 4 shows the data for concentration of eNOS in lung tissues expressed as percentage of Vehicle. Monocrotaline caused a significant

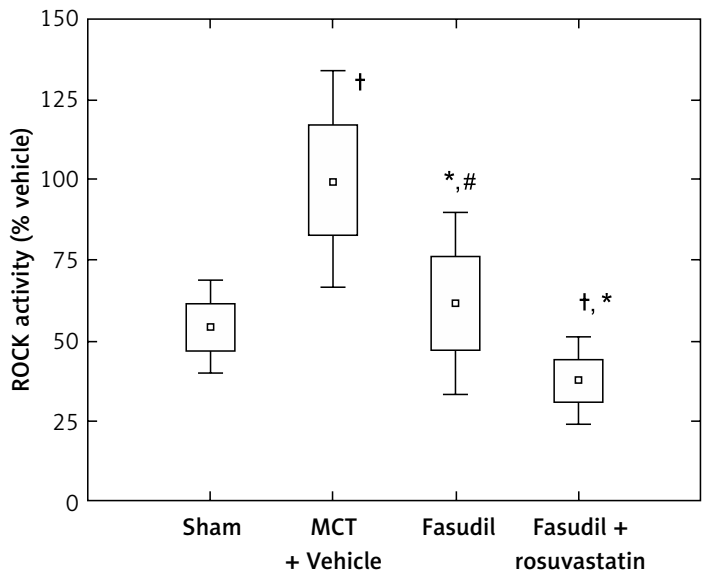

Figure 3. Comparison of ROCK activity of different animal groups with induced pulmonary hypertension, as percentage of MCT + Vehicle. Sham $(n=7)$; MCT + Vehicle $(n=9)$; fasudil $(n=6)$; fasudil + rosuvastatin $(n=9)$

The histograms indicate: mean $\pm S D(\square)$ and mean \pm $1.96{ }^{*} S D(\perp) ;{ }^{\dagger} p<0.05$ vs. Sham; ${ }^{*} p<0.0005$ vs. MCT + Vehicle; $" p<0.005$ vs. combination group. 


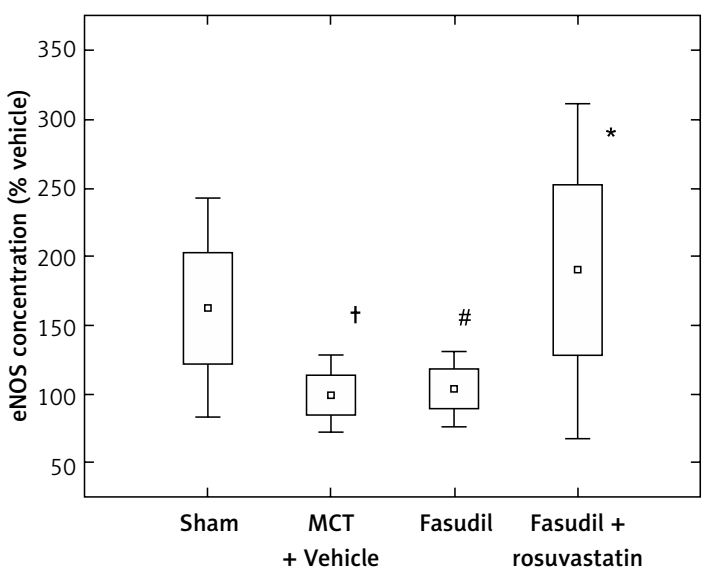

Figure 4. Comparison of eNOS level in different animal groups with induced pulmonary hypertension, as percentage of MCT + Vehicle. Sham $(n=8)$; MCT + Vehicle $(n=8)$; fasudil $(n=6)$; fasudil + rosuvas$\operatorname{tatin}(n=8)$

The histograms indicate: mean $\pm S D$ ( $\square$ ) and mean $\pm 1.96^{*} S D$ $(\perp) ;{ }^{\dagger} p<0.05$ vs. Sham; ${ }^{*} p<0.05$ vs. MCT + Vehicle; ${ }^{*} p<0.05$ vs. combination group.

decrease of eNOS compared to Sham $(p<0.05)$. 14-day administration of fasudil with rosuvastatin resulted in an increase of eNOS compared to MCT + Vehicle $(p<0.05)$ or fasudil alone $(p<0.05)$.

\section{Discussion}

Statins have already been investigated in a variety of experimental pulmonary hypertension models. It has been shown previously that simvastatin both prevents and regresses pulmonary hypertension with right ventricle hypertrophy, and vascular remodeling in rats with hypoxia-induced $\mathrm{PH}$, as well as in an MCT model [26-28]. The benefits of statins given in combination with a conventional regimen have also been investigated in several experimental studies, mainly concerning lipophilic statins, such as simvastatin or atorvastatin, and the phosphodiesterase type 5 (PDE-5) inhibitor sildenafil. These beneficial changes included both hemodynamic effects, i.e. further decreases in right ventricle systolic pressure (RVSP), right ventricle hypertrophy (RVH), pulmonary artery smooth muscle cell (PASMC) proliferation and vascular remodeling, as well as molecular effects such as elevation of cyclic guanosine monophosphate (cGMP) $[18,19]$. The recent studies indicate that also hydrophilic rosuvastatin might exert some beneficial impact on pulmonary hypertension [29].

In our previous study we demonstrated rosuvastatin to exert a moderate impact on hemodynamics and RV hypertrophy in rats with MCT-induced $\mathrm{PH}$. The mean RVP values $(47.7 \mathrm{~mm} \mathrm{Hg}$ ) observed in MCT-induced rats receiving rosuvastatin at $10 \mathrm{mg} / \mathrm{kg}$ for 14 days were only $26.5 \%$ lower than those receiving the vehicle on day 29 of the experiment [22].

Thus the aim of the present experiments was to assess whether rosuvastatin intensifies the effects of fasudil therapy rather than improving $\mathrm{PH}$ itself. As with Jiang et al. [30], who observed the effects of a medium (10 mg/ $\mathrm{kg})$ and a high (30 mg/kg) oral dose of fasudil on significant reduction of mPAP, 14-day administration of fasudil alone at $15 \mathrm{mg} / \mathrm{kg}$ in the present study reversed the MCT-induced increase in RVP and myocardial hypertrophy. Moreover, the present observations indicate further significant improvement of the examined parameters when rosuvastatin was added to ROCK inhibitor, and it can be hypothesized that the observed effects could be linked to the Rho/Rho-kinase pathway.

Several studies refer to changes of RhoA activity in humans. For example, Guilluy et al. [4] found the lungs, platelets, and pulmonary artery smooth muscle cells from patients with idiopathic pulmonary hypertension to be characterized by marked elevation in RhoA and Rho kinase activities.

Similar to previous reports showing that expression of ROCK proteins can be significantly up-regulated in monocrotaline-induced $\mathrm{PH}[5,30]$, in the present study MCT injection caused a significant increase in ROCK activity. ROCK activity in isolated lung tissues obtained from the group receiving fasudil alone was decreased. This is in agreement with the study by Jiang et al. [30], who denoted suppression of Rho-kinase in pulmonary arteries, but not in the aorta, as a result of oral administration of fasudil to MCT-induced $\mathrm{PH}$ rats. Moreover, we have observed the further, significant decrease of ROCK activity, as a result of chronic combined administration of fasudil with rosuvastatin. We suggest that such alterations in the RhoA/ROCK pathway may, at least partially, contribute to significant improvement of RVP and $\mathrm{RV}$ hypertrophy in the group receiving fasudil with statin, compared to fasudil alone.

To our knowledge this is the first study demonstrating such effects of statin administration in MCT-induced $\mathrm{PH}$. A very recent study by Naraoka et al. [23] described the synergism of concurrent administration of another statin, pitavastatin, and the Rho kinase inhibitor fasudil. However, the study aimed to assess their effects on another disease: subarachnoid hemorrhage (SAH). The examined agents given together effectively prevented cerebral vasospasm in rabbit. Similar to the results obtained in the present study, the authors reported significant inhibition of Rho-kinase expression by combined treatment compared to fasudil alone. They concluded that such reduction of ROCK may result from both the direct inhibitory effect of fasudil on Rho-kinase and the depression of RhoA activity by statin [31]. The RhoA/ROCK pathway 
is involved in diverse cellular functions, including SMC contraction, cytoskeletal rearrangement, cell migration, cell proliferation and gene expression. Evidence strongly suggests that small GTPase RhoA and its downstream effector, ROCK, play a role in $\mathrm{PH}$ and PASMC proliferation. RhoA was demonstrated to be mediated by vasoconstrictors such as angiotensin II, endothelin-1 or serotonin [32]. The role of RhoA/ROCK signaling in $\mathrm{PH}$ development in MCT rats has also been confirmed [5].

Moreover, the successful results of Rho-kinase inhibitors in several animal models of $\mathrm{PH}$ include improvement of hemodynamic parameters and pulmonary vascular remodeling, enhancement of vascular smooth muscle cell (VSMC) apoptosis or amelioration of endothelial dysfunction [3, 12, 13].

Statin pleiotropy in $\mathrm{PAH}$ may be at least partly attributed to the reduced posttranslational prenylation of the Ras, Rho and Rac proteins, and the inhibition of the RhoA/Rho-kinase pathway. As mentioned above, statins have been shown to inhibit RhoA [33]. It has been reported that statin inhibition of an early step in the cholesterol biosynthetic pathway leads to inhibition of the synthesis of isoprenoids such as farnesyl pyrophosphate and geranylgeranyl pyrophosphate, which are important posttranslational lipid attachments for intracellular signaling molecules, including Rho GTPases. Inactivation of the Rho/Rho kinase pathway might play a major role in statin-induced increases in endothelial function. Zhao et al. [18], in a study on a rat model of hypoxia-induced pulmonary hypertension, demonstrated that simvastatin attenuates GTP-RhoA expression in the lung and right ventricle during exposure to hypoxia, and this effect is accompanied by a significant lowering of pulmonary artery pressure and reduction in right ventricular hypertrophy.

The question is whether the additional effect of rosuvastatin on ROCK signaling is crucial for the $\mathrm{PAH}$ reversal observed in the present study, especially since fasudil already inhibits ROCK. It cannot be excluded that the further PAH improvement observed in the combination group may be a result of the impact of statin on the ROCK or other pathways. The use of statins in treating PAH may have beneficial effects on angiogenesis, atherosclerosis or inflammation, and influence the suppression of angiotensin II/endothelin-1-induced release of matrix metalloproteinase or reduce the secretion of interleukin-6 (IL-6) and monocyte chemoattractant protein-1 (MCP-1) in PASMC [34]. Statin-elicited increases in endothelial nitric oxide production have been shown to play a key role in vascular reactivity, as well as the modulation of smooth muscle cell proliferation or cellular inflammation [16].

In experimental studies statin was shown to attenuate monocrotaline-induced $\mathrm{PH}$ also via regulation of Akt/eNOS signaling [28, 35]. Statin activates Akt, which upregulates the activity of eNOS and enhances NO release from endothelial cells [23], and this effect is a consequence of the statin-mediated inhibition of geranylgeranylation of the small G-protein Rho. Reduced production of nitric oxide relates to endothelial dysfunction in $\mathrm{PAH}$. It has been speculated that restoration of eNOS expression by statin might be associated with the down-regulation of ROCK expression [29] and contribute to the protective role of HMG-CoA reductase inhibitors in disease development. In the present study, the level of eNOS in lung tissues was reduced in rats exposed to MCT compared to those exposed to saline. Despite quite large variability being observed, significantly higher levels of eNOS were observed in rats receiving combined treatment than those administered with Vehicle or fasudil alone. At the very least, this may suggest a tendency toward more pronounced NO signaling as a result of combining statin with ROCK inhibitor. However, such significant enhancement of eNOS was not seen in the fasudil group, and this observation is consistent with the results obtained by Naraoka in an SAH model; fasudil given in monotherapy did not result in an eNOS increase [23]. The previous experimental studies on pulmonary hypertension in rats showed fasudil-related improvement of eNOS expression, but the administered doses were higher (30 and $100 \mathrm{mg} / \mathrm{kg}$ bw) [12, 30] compared to the present study $(15 \mathrm{mg} / \mathrm{kg} \mathrm{bw})$. On the other hand, such a low dose of fasudil as that used in our experiment seems to correspond with clinical applications in future. Plasma concentrations of fasudil/hydroxyfasudil that were achieved by the similarly low dose of fasudil $(10 \mathrm{mg} / \mathrm{kg})$ in a study by Jiang et al. [30] were within their clinical therapeutic range in humans [33].

Moreover, such interaction between ROCK inhibitor and statin seems to be clinically relevant, as it provides the possibility of reducing the dose of fasudil and thereby diminishing the risk of systemic vasodilatation.

There are some limitations of the study. Considering the above-mentioned aspects, the monocrotaline model might not fully represent pulmonary hypertension in humans, and the effectiveness of combination therapy should be evaluated in other $\mathrm{PH}$ models with different etiologies. Secondly, the additional measurements of cardiac output, as well as cardiac contractility assessed by echocardiogram, could provide valuable supplementary information regarding the influence of the examined agents on hemodynamics and cardiac capacity. The decreased pulse pressure amplitude observed in the rosuvastatin + fasudil group might suggest lower stroke vol- 
ume and reduced left ventricular contractility. In our opinion, there is a faint possibility that such a therapeutic intervention mainly reduces cardiac contractility, with a lower impact on pulmonary circulation. Previous reports do not indicate that statin or fasudil has any detrimental impact on the decrease of cardiac output [36, 37]. However, such an assumption should be confirmed. Thirdly, effects on pulmonary vessels could demonstrate the antiremodeling properties of proposed regimens, in addition to the hemodynamics and RV hypertrophy results.

In conclusion, combination treatment could extensively reverse $\mathrm{PH}$ due to the beneficial impact of rosuvastatin on the enhancement of eNOS and ROCK activity, and such a combination may represent an option for the treatment of pulmonary arterial hypertension. However, further studies are needed to evaluate the precise mechanistic background of such drug-drug interaction.

\section{Acknowledgments}

The study was supported by the Medical University of Lodz, grant no. 502-03/3-011-02/50234-028;503/3-011-02/503-31-002,503/3-011-02/ 503-06-300.

\section{Conflict of interest}

The authors declare no conflict of interest.

\section{References}

1. Farber HW, Loscalzo J. Pulmonary arterial hypertension. N Engl J Med 2004; 351: 1655-65.

2. Morrell NW, Adnot S, Archer SL, et al. Cellular and molecular basis of pulmonary arterial hypertension. J Am Coll Cardiol 2009; 54: S20-31.

3. Nagaoka T, Gebb SA, Karoor V, et al. Involvement of RhoA/Rho kinase signaling in pulmonary hypertension of the fawn-hooded rat. J Appl Physiol 2006; 100: 9961002.

4. Guilluy C, Eddahibi S, Agard C, et al. RhoA and Rho kinase activation in human pulmonary hypertension: role of 5-HT signaling. Am J Respir Crit Care Med 2009; 179: 1151-8.

5. Li X, Peng HJ, Tan N, et al. Involvement of asymmetric dimethylarginine and Rho kinase in the vascular remodeling in monocrotaline-induced pulmonary hypertension. Vascul Pharmacol 2010; 53: 223-9.

6. Wilson DW, Segall HJ, Pan LC, Lamé MW, Estep JE, Morin D. Mechanisms and pathology of monocrotaline pulmonary toxicity. Crit Rev Toxicol 1992; 22: 307-5.

7. Zambelli V, Santaniello A, Fumagalli F, et al. Efficacy of aminaftone in a rat model of monocrotaline-induced pulmonary hypertension. Eur J Pharmacol 2011; 667: 287-91.

8. Li F, Xia W, Li A, Zhao C, Sun R. Long-term inhibition of Rho kinase with fasudil attenuates high flow induced pulmonary artery remodeling in rats. Pharmacol Res 2007; 55: 64-71.

9. Fukumoto $\mathrm{Y}$, Yamada N, Matsubara $\mathrm{H}$, et al. Doubleblind, placebo-controlled clinical trial with a rho-kinase inhibitor in pulmonary arterial hypertension. Circ J 2013; 77: 2619-25.

10. Ishikura K, Yamada N, Ito $M$, et al. Beneficial acute effects of rho-kinase inhibitor in patients with pulmonary arterial hypertension. Circ J 2006; 70: 174-8.

11. Fujita H, Fukumoto Y, Saji K, et al. Acute vasodilator effects of inhaled fasudil, a specific Rho-kinase inhibitor, in patients with pulmonary arterial hypertension. Heart Vessels 2010; 25: 144-9.

12. Abe K, Tawara S, Oi K, et al. Long-term inhibition of Rho-kinase ameliorates hypoxia-induced pulmonary hypertension in mice. J Cardiovasc Pharmacol 2006; 48: 280-5.

13. Abe K, Shimokawa H, Morikawa K, et al. Long-term treatment with a Rho-kinase inhibitor improves monocrotaline-induced fatal pulmonary hypertension in rats. Circ Res 2004; 94: 385-93.

14. Fukumoto $\mathrm{Y}$, Yamada N, Matsubara H, et al. Doubleblind, placebo-controlled clinical trial with a rho-kinase inhibitor in pulmonary arterial hypertension. Circ J 2013; 77: 2619-25.

15. Rikitake Y, Kim HH, Huang Z, et al. Inhibition of Rho kinase (ROCK) leads to increased cerebral blood flow and stroke protection. Stroke 2005; 36: 2251-7.

16. Liao JK, Laufs U. Pleiotropic effects of statins. Annu Rev Pharmacol Toxicol 2005; 45: 89-118.

17. Wolfrum S, Jensen KS, Liao JK. Endothelium-dependent effects of statins. Arterioscler Thromb Vasc Biol 2003; 23: 729-36.

18. Zhao L, Sebkhi A, Ali O, et al. Simvastatin and sildenafil combine to attenuate pulmonary hypertension. Eur Respir J 2009; 34: 948-57.

19. Kuang T, Wang J, Pang B, et al. Combination of sildenafil and simvastatin ameliorates monocrotaline-induced pulmonary hypertension in rats. Pulm Pharmacol Ther 2010; 23: 456-64.

20. Zeng WJ, Xiong CM, Zhao L, et al. Atorvastatin in pulmonary arterial hypertension (APATH) study. Eur Respir J 2012; 40: 67-74

21. Wilkins MR, Ali O, Bradlow W, et al. Simvastatin as a treatment for pulmonary hypertension trial. Am J Respir Crit Care Med 2010; 181: 1106-13.

22. Jasińska-Stroschein M, Owczarek J, Surowiecka A, Kącikowska J, Orszulak-Michalak D. HMG-CoA reductase inhibitors: an opportunity for the improvement of imatinib safety. An experimental study in rat pulmonary hypertension. Pharmacol Rep 2015; 67: 32-7.

23. Naraoka M, Munakata A, Matsuda N, Shimamura N, Ohkuma H. Suppression of the Rho/Rho-kinase pathway and prevention of cerebral vasospasm by combination treatment with statin and fasudil after subarachnoid hemorrhage in rabbit. Transl Stroke Res 2013; 4: 368-74.

24. Schermuly RT, Kreisselmeier KP, Ghofrani HA, et al. Antiremodeling effects of iloprost and the dual-selective phosphodiesterase 3/4 inhibitor tolafentrine in chronic experimental pulmonary hypertension. Circ Res 2004; 94: 1101-8.

25. Jasińska-Stroschein M, Owczarek J, Łuczak A, OrszulakMichalak D. The beneficial impact of fasudil and sildenafil on monocrotaline-induced pulmonary hypertension in rats: a hemodynamic and biochemical study. Pharmacology 2013; 91: 178-84.

26. Girgis RE, Li D, Zhan X et al. Attenuation of chronic hypoxic pulmonary hypertension by simvastatin. Am J Physiol Heart Circ Physiol 2003; 285: H938-45.

27. Nishimura T, Vaszar LT, Faul JL, et al. Simvastatin rescues rats from fatal pulmonary hypertension by inducing 
apoptosis of neointimal smooth muscle cells. Circulation 2003; 108: 1640-5.

28. Pei Y, Ma P, Wang X, et al. Rosuvastatin attenuates monocrotaline-induced pulmonary hypertension via regulation of Akt/eNOS signaling and asymmetric dimethylarginine metabolism. Eur J Pharmacol 2011; 666: 165-72.

29. Li XL, Guan RJ, Li JJ. Attenuation of monocrotaline-induced pulmonary arterial hypertension in rats by rosuvastatin. J Cardiovasc Pharmacol 2012; 60: 219-26.

30. Jiang BH, Tawara S, Abe K, Takaki A, Fukumoto Y, Shimokawa $H$. Acute vasodilator effect of fasudil, a Rho-kinase inhibitor, in monocrotaline-induced pulmonary hypertension in rats. J Cardiovasc Pharmacol 2007; 49: 85-9.

31. Rashid M, Tawara S, Fukumoto Y, Seto M, Yano K, Shimokawa $\mathrm{H}$. Importance of Rac1 signaling pathway inhibition in the pleiotropic effects of HMG-CoA reductase inhibitors. Circ J 2009; 73: 361-70.

32. Homma N, Nagaoka T, Karoor V, et al. Involvement of RhoA/Rho kinase signaling in protection against monocrotaline-induced pulmonary hypertension in pneumonectomized rats by dehydroepiandrosterone. Am J Physiol Lung Cell Mol Physiol 2008; 295: L71-8.

33. Masumoto A, Mohri M, Shimokawa H, Urakami L, Usui M, Takeshita A. Suppression of coronary artery spasm by the Rho-kinase inhibitor fasudil in patients with vasospastic angina. Circulation 2002; 105: 1545-7.

34. Katsiki N, Wierzbicki AS, Mikhailidis DP. Pulmonary arterial hypertension and statins: an update. Curr Opin Cardiol 2011; 26: 322-6.

35. Sun X, Ku DD. Rosuvastatin provides pleiotropic protection against pulmonary hypertension, right ventricular hypertrophy, and coronary endothelial dysfunction in rats. Am J Physiol Heart Circ Physiol 2008; 294: H801-9.

36. Zhang L, Zhang S, Jiang H, Sun A, Zou Y, Ge J. Effects of statin treatment on cardiac function in patients with chronic heart failure: a meta-analysis of randomized controlled trials. Clin Cardiol 2011; 34: 117-23.

37. Oka M, Homma N, Taraseviciene-Stewart L, et al. Rho kinase-mediated vasoconstriction is important in severe occlusive pulmonary arterial hypertension in rats. Circ Res 2007; 100: 923-9. 